\title{
Assessment of evaluated $(n, d)$ energy-angle elastic scattering distributions using MCNP simulations of critical measurements and simplified calculation benchmarks
}

\author{
K.S. Kozier \\ Atomic Energy of Canada Limited, Chalk River Laboratories, Chalk River, Ontario, Canada, K0J 1J0
}

\begin{abstract}
Different evaluated (n,d) energy-angle elastic scattering distributions produce k-effective differences in MCNP5 simulations of critical experiments involving heavy water $\left(\mathrm{D}_{2} \mathrm{O}\right)$ of sufficient magnitude to suggest a need for new (n,d) scattering measurements and/or distributions derived from modern theoretical nuclear models, especially at neutron energies below a few $\mathrm{MeV}$. The present work focuses on the small reactivity change of $<1 \mathrm{mk}$ that is observed in the MCNP5 $\mathrm{D}_{2} \mathrm{O}$ coolant-void-reactivity calculation bias for simulations of two pairs of critical experiments performed in the ZED-2 reactor at the Chalk River Laboratories when different nuclear data libraries are used for deuterium. The deuterium data libraries tested include ENDF/B-VII.0, ENDF/B-VI.4, JENDL-3.3 and a new evaluation, labelled Bonn-B, which is based on recent theoretical nuclear-model calculations. Comparison calculations were also performed for a simplified, two-region, spherical model having an inner, 250-cm radius, homogeneous sphere of $\mathrm{UO}_{2}$, without and with deuterium, and an outer 20-cm-thick deuterium reflector.
\end{abstract}

\section{Introduction}

During testing of the final release of version VI of the Evaluated Nuclear Data File (ENDF/B-VI.8), Los Alamos National Laboratory (LANL) staff discovered [1] that the calculated eigenvalues $\left(k_{\text {eff }}\right)$ for a series of Highly Enriched Uranium (HEU) heavy-water $\left(\mathrm{D}_{2} \mathrm{O}\right)$ solution critical benchmark experiments had decreased by about $10 \mathrm{mk}(1 \mathrm{mk}=$ a change in $k_{\text {eff }}$ of 0.001 ) relative to results obtained with releases VI.0 to VI.4 for deuterium $\left({ }^{2} \mathrm{H}\right.$ or D). In contrast, only small reactivity differences $(<1 \mathrm{mk})$ were observed [2] when similar substitutions of the ${ }^{2} \mathrm{H}$ nuclear data were made in MCNP5 ${ }^{\mathrm{TM}}{ }_{1}$ [3] (Monte Carlo N-Particle) simulations of critical measurements involving low-leakage, heterogeneous lattices of natural-uranium (NU) fuel rods immersed in $\mathrm{D}_{2} \mathrm{O}$ moderator in the ZED-2 (Zero Energy Deuterium) reactor at the Chalk River Laboratories (CRL). Subsequent investigations [4] using a simplified, two-region, spherical benchmark model, consisting of an inner sphere of $U$ metal surrounded by a ${ }^{2} \mathrm{H}$ reflector, demonstrated qualitatively that this difference in reactivity sensitivity is mainly due to the difference in $\mathrm{U}$ enrichment between the LANL and CRL experiments.

However, CRL Coolant Void Reactivity (CVR) experiments involving pairs of experiments with air (i.e., void) and $\mathrm{D}_{2} \mathrm{O}$ as coolant showed a small $(<1 \mathrm{mk})$, but significant, sensitivity to the ${ }^{2} \mathrm{H}$ nuclear data files in calculations of the MCNP5 CVR simulation bias (defined here as the difference between the $k_{\text {eff }}$ bias (i.e., calculated $k_{\text {eff }}-1.0$ ) for the aircooled and $\mathrm{D}_{2} \mathrm{O}$-cooled critical states).

The present work focuses on the sensitivity of the ZED-2 MCNP5 CVR calculation bias to the nuclear data libraries used for ${ }^{2} \mathrm{H}$. It explores the cause of the reactivity effect by examining its detailed neutron-energy dependence and by comparing with results for a simplified calculation benchmark

\footnotetext{
${ }^{1}$ MCNP is a trademark of the Regents of the University of California, Los Alamos National Laboratory.
}

model consisting of an inner, $250-\mathrm{cm}$ radius, homogeneous sphere of $\mathrm{UO}_{2}$ surrounded by an outer 20 -cm-thick ${ }^{2} \mathrm{H}$ reflector. The benchmark simulations are performed without and with ${ }^{2} \mathrm{H}$ (up to the concentration corresponding to ${ }^{2} \mathrm{H}$ in $\mathrm{D}_{2} \mathrm{O}$ ) mixed homogeneously with the $\mathrm{UO}_{2}$, to mimic the basic neutronic effects of $\mathrm{D}_{2} \mathrm{O}$ coolant voiding.

For both the ZED-2 and spherical benchmark simulations, MCNP5 results are compared for deuterium nuclear data libraries from ENDF/B-VII.0, ENDF/B-VI.4, JENDL-3.3 (Japanese Evaluated Nuclear Data Library) and a preliminary new evaluation, labelled Bonn-B, which is based on recent theoretical nuclear-model calculations [5].

\section{Method}

The MCNP5 calculations were performed using version 1.40. The ZED-2 results are based on 2100 cycles of 60,000 source neutrons, skipping the first 100 cycles. The spherical benchmark model results used 1500 cycles of 60,000 neutrons, skipping the first 500 cycles.

The ZED-2 simulations correspond to two pairs of $\mathrm{D}_{2} \mathrm{O}$ and air-cooled lattices at room temperature, consisting of an inner reactor core zone of 55 fuel channels (assemblies of concentric aluminium tubes that mock up corresponding arrangements of CANDU ${ }^{\circledR} 2$ zirconium-alloy pressure tubes and calandria tubes) containing vertical stacks of five fuel bundles of 28-element $\mathrm{NU} \mathrm{UO}_{2}$ fuel, similar to the fuel bundles used in the Pickering CANDU power reactors. The lattice configurations used a triangular lattice pitch of $31.0 \mathrm{~cm}$ that is approximately equivalent neutronically to a standard CANDU square-lattice pitch of $28.575 \mathrm{~cm}$.

The pairs of lattices differ primarily in having an outer zone of 30 "booster" rods of different fuel types. One pair

${ }^{2}$ CANDU $^{\circledR}$ (Canada Deuterium Uranium) is a registered trademark of Atomic Energy of Canada Limited (AECL). 
Table 1. MCNP5 $k_{\text {eff }}$ bias results for ZED-2 simulations (in mk).

\begin{tabular}{lccccc}
\hline \multirow{2}{*}{$\begin{array}{l}\text { Booster } \\
\text { rods }\end{array}$} & $\begin{array}{c}\text { 28-el. } \\
\mathrm{UO}_{2}\end{array}$ & ENDF/ & ENDF/ & JENDL- & Bonn-B \\
& coolant & B-VII.0 & B-VI.4 & 3.3 & \\
\hline \multirow{4}{*}{ ZEEP } & Air & 3.237 & 3.396 & 2.838 & 3.434 \\
& & \pm 0.049 & \pm 0.049 & \pm 0.048 & \pm 0.048 \\
& $\mathrm{D}_{2} \mathrm{O}$ & 2.685 & 2.492 & 1.884 & 2.757 \\
& & \pm 0.048 & \pm 0.049 & \pm 0.047 & \pm 0.047 \\
\hline \multirow{4}{*}{ 19-el. } & Air & 2.941 & 3.019 & 2.639 & 3.169 \\
& & \pm 0.048 & \pm 0.047 & \pm 0.049 & \pm 0.049 \\
& $\mathrm{D}_{2} \mathrm{O}$ & 2.174 & 1.881 & 1.187 & 2.119 \\
& & \pm 0.047 & \pm 0.048 & \pm 0.048 & \pm 0.048 \\
\hline
\end{tabular}

uses ZEEP (Zero Energy Experimental Pile) rods, consisting of a single, large $(3.26-\mathrm{cm}$ diameter) NU metal rod clad in aluminium. The second pair uses 19-element, NU-metal assemblies with $\mathrm{D}_{2} \mathrm{O}$ coolant in aluminium coolant tubes. The experimental error in a ZED-2 critical configuration is estimated to be $\pm 0.2 \mathrm{mk}$ and is dominated by the uncertainty in $\mathrm{D}_{2} \mathrm{O}$ purity (i.e., degree of contamination with $\mathrm{H}_{2} \mathrm{O}$ ).

The ENDF/B library recently underwent a major revision with the release of version VII.0 [6]. Accordingly, this work was updated to use ACE (A Compact ENDF format) files for all nuclides from ENDF/B-VII.0 (specifically those prepared by the National Nuclear Data Center (NNDC) and distributed by the Radiation Shielding Information Computational Center (RSICC)) as the reference case.

The ENDF/B-VII.0 library includes features that are not available in ENDF/B-VI, specifically thermal scattering data for $\mathrm{O}$ in $\mathrm{UO}_{2}$ and $\mathrm{U}$ in $\mathrm{UO}_{2}$. The former has a significant impact on the calculated ZED-2 MCNP5 CVR bias, reducing it consistently by about $0.4 \mathrm{mk}$ relative to earlier work [2] (and increasing the $k_{\text {eff }}$ values by 0.7 to $2.7 \mathrm{mk}$ ). Accordingly, all MCNP5 cases used the thermal scattering data for $\mathrm{UO}_{2}$. Moreover, it was decided to use $\mathrm{UO}_{2}$ in the comparison calculations for a spherical system so as to be more consistent with the ZED-2 28-element $\mathrm{UO}_{2}$ fuel.

The (n,d) energy-angle probability distribution in the ENDF/B-VII.0 ${ }^{2} \mathrm{H}$ nuclear data library is the same in all versions released since ENDF/B-VI.5. It is based on a coupledchannels R-matrix analysis that was revised at incident neutron energies $<3.2 \mathrm{MeV}$ to improve agreement with experiment. Perturbation calculations were performed relative to the ENDF/B-VII.0 reference cases by changing only the ${ }^{2} \mathrm{H}$ ACE data file to:

- ENDF/B-VI.4, using an ACE file prepared at LANL. The $(n, d)$ energy-angle probability distribution data are identical for ENDF/B-VI.0 through VI.4.

- JENDL-3.3, using an ACE file obtained from RSICC. These data are derived from theoretical calculations based on solution of the Faddeev equation. Bonn-B, a new determination [5] for (n,d) scattering over the energy range from $50 \mathrm{keV}$ to $10 \mathrm{MeV}$ based on solving the AltGrassberger-Sandhas equation. The corresponding ACE file was obtained by inserting the Bonn-B total scattering and energy-angle probability distribution data into the
Table 2. MCNP5 CVR bias results for ZED-2 simulations (in mk).

\begin{tabular}{lcccc}
\hline $\begin{array}{l}\text { Booster } \\
\text { rods }\end{array}$ & ENDF/ & ENDF/ & JENDL- & Bonn-B \\
ZEEP & 0.552 & 0.904 & 0.954 & 0.677 \\
& \pm 0.068 & \pm 0.070 & \pm 0.069 & \pm 0.068 \\
\hline 19-el. & 0.767 & 1.138 & 1.452 & 1.050 \\
& \pm 0.067 & \pm 0.067 & \pm 0.066 & \pm 0.069 \\
\hline Average & 0.66 & 1.02 & 1.02 & 0.86 \\
\hline
\end{tabular}

ENDF/B-VII.0 file and processing the modified file with NJOY-99.0 [7].

The various ${ }^{2} \mathrm{H}$ nuclear data libraries differ mainly in the $(\mathrm{n}, \mathrm{d})$ energy-angle scattering probability distributions for neutron energies <3.2 MeV. Recent direct comparisons [5] show nonphysical irregularities in the ENDF/B-VII.0 data, which suggest they may be in need of revision. The alternate ${ }^{2} \mathrm{H}$ data libraries typically show a higher probability for backscattering, particularly near $180^{\circ}$ in the centre-of-mass reference frame. Additionally, a review of the experimental data that form the basis for the ENDF/B-VII. $0{ }^{2} \mathrm{H}$ evaluation identified inconsistencies that suggest a need for new $(n, d)$ scattering measurements in this energy range.

For the simplified benchmark calculations, the inner sphere radius of $250 \mathrm{~cm}$ yields a neutron leakage roughly comparable to ZED-2. Calculations were performed at ${ }^{2} \mathrm{H}$ concentrations corresponding to $0,10,50$ and $100 \%$ of the number density for ${ }^{2} \mathrm{H}$ in $\mathrm{D}_{2} \mathrm{O}$. The ${ }^{2} \mathrm{H}$ number density in the $20-\mathrm{cm}$-thick reflector was fixed at $100 \%{ }^{2} \mathrm{H}$. The thermal scattering data for $\mathrm{D}$ in $\mathrm{D}_{2} \mathrm{O}, \mathrm{O}$ in $\mathrm{UO}_{2}$ and $\mathrm{U}$ in $\mathrm{UO}_{2}$ were used throughout for consistency with the ZED-2 calculations.

\section{Results}

Table 1 shows the MCNP5 $k_{\text {eff }}$ bias results for the ZED2 simulations along with the $(1 \sigma)$ statistical uncertainties. In every case, MCNP5 overestimates criticality by a small amount, ranging from 1.2 to $3.4 \mathrm{mk}$. Since the $k_{\text {eff }}$ bias is

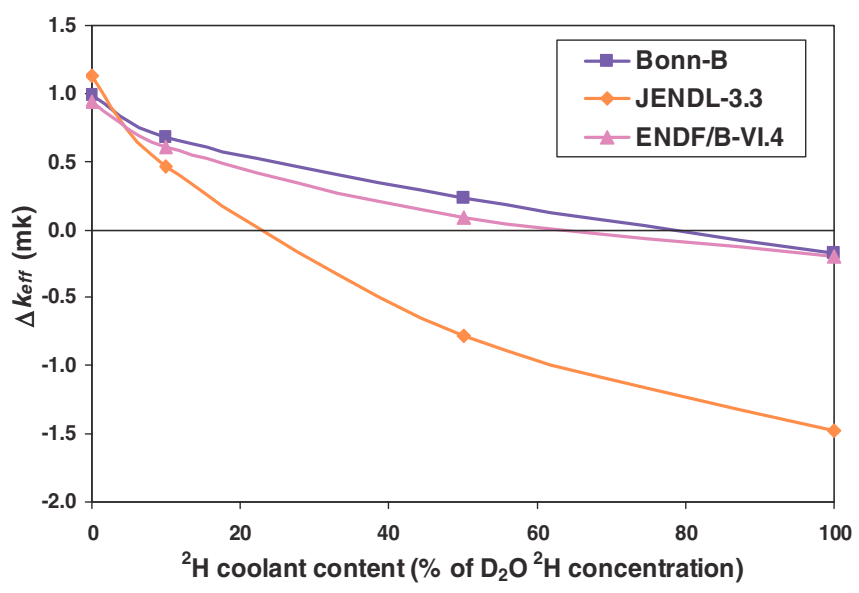

Fig. 1. $\Delta k_{\text {eff }}$ relative to ENDF/B-VII.0 for alternate ${ }^{2} \mathrm{H}$ data libraries as a function of ${ }^{2} \mathrm{H}$ content for spherical $\mathrm{UO}_{2}$ benchmark. 


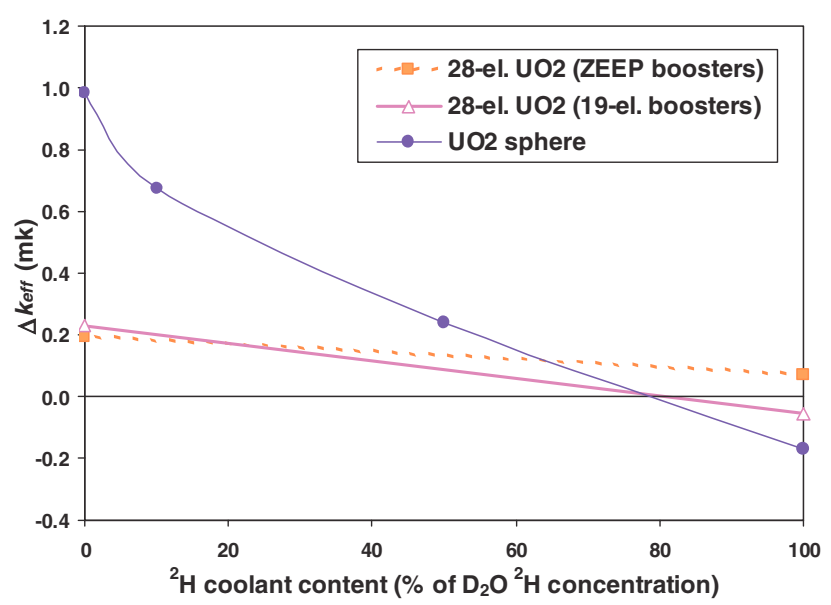

Fig. 2. $\Delta k_{e f f}$ sensitivity of Bonn-B results relative to ENDF/B-VII.0 for $\mathrm{UO}_{2}$ sphere and ZED-2 28element $\mathrm{UO}_{2} \mathrm{CVR}$ experiments.

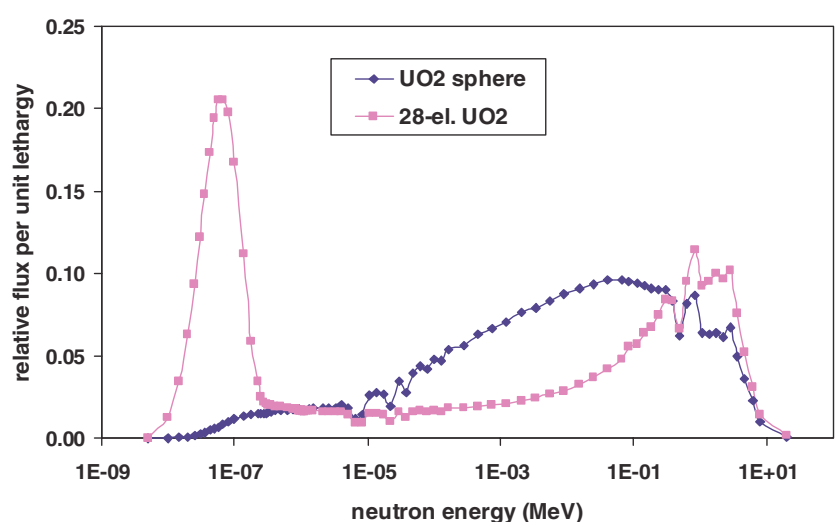

Fig. 3. Comparison of calculated flux spectra for $\mathrm{UO}_{2}$ sphere and 28-element $\mathrm{UO}_{2}$ fuel $\left(100 \%{ }^{2} \mathrm{H}\right.$; Bonn-B; ZEEP boosters).

always larger for the air-cooled lattice configurations relative to the $\mathrm{D}_{2} \mathrm{O}$-cooled cases, a small, positive, systematic CVR calculation bias is observed, as shown in table 2 .

The last line of table 2 gives average CVR bias values for each of the ${ }^{2} \mathrm{H}$ data libraries. The Bonn-B average CVR bias is $0.86 \mathrm{mk}$, only $0.2 \mathrm{mk}$ larger than the lowest value of $0.66 \mathrm{mk}$, which is obtained with the reference ENDF/B-VII. $0{ }^{2} \mathrm{H}$ data library.

The spherical benchmark system is subcritical, with calculated $k_{\text {eff }}$ values ranging from about 0.387 (the minimum occurs near $\left.10 \%{ }^{2} \mathrm{H}\right)$ to about $0.457\left(100 \%{ }^{2} \mathrm{H}\right)$. The reactivity sensitivity was assessed by calculating the $\Delta k_{\text {eff }}$ with respect to the reference ENDF/B-VII.0 results as a function of ${ }^{2} \mathrm{H}$ content. These results are shown in figure 1 . The uncertainties of the data points are $< \pm 0.07 \mathrm{mk}$.

Figure 1 qualitatively confirms the behaviour observed in earlier reactivity sensitivity studies [2] for the ZED2 28element $\mathrm{UO}_{2} \mathrm{CVR}$ experiments, where the $\Delta k_{\text {eff }}$ value was positive for air coolant (i.e., $0 \%{ }^{2} \mathrm{H}$ ) when ENDF/B-VI.4 was used for ${ }^{2} \mathrm{H}$ instead of ENDF/B-VIIbeta1, and negative for $\mathrm{D}_{2} \mathrm{O}$ coolant (i.e., $100 \%{ }^{2} \mathrm{H}$ ). Figure 1 also shows that Bonn$\mathrm{B}$ and ENDF/B-VI.4 yield similar reactivity results, which is consistent with the similar ZED-2 CVR bias values obtained for these ${ }^{2} \mathrm{H}$ libraries in table 2 .

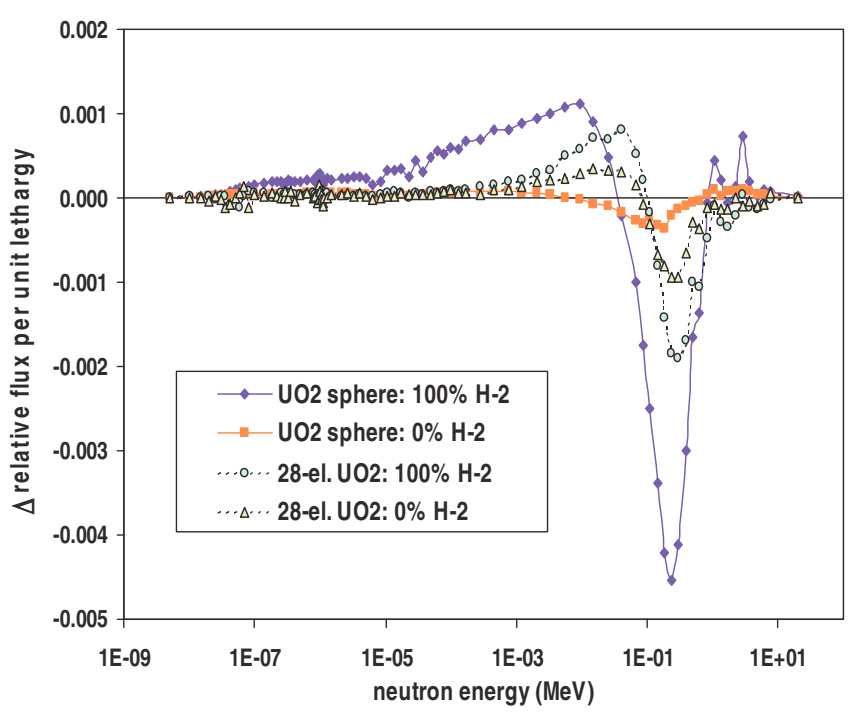

Fig. 4. Difference in flux spectra using Bonn-B and ENDF/B-VII.0 ${ }^{2} \mathrm{H}$ data for $\mathrm{UO}_{2}$ sphere and 28-element $\mathrm{UO}_{2}$ at 0 and $100 \%{ }^{2} \mathrm{H}$.

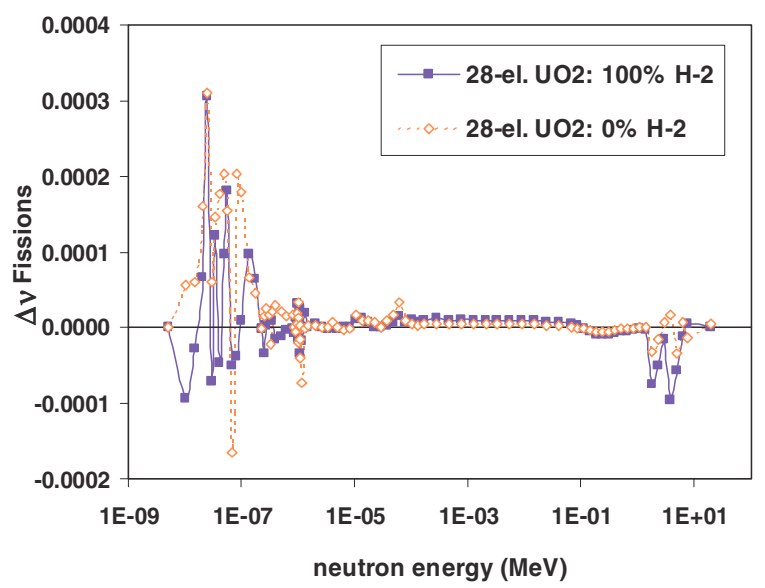

Fig. 5. Change in fission neutron yields between Bonn- $\mathrm{B}$ and ENDF/B-VII. ${ }^{2} \mathrm{H}$ data for 28 -element $\mathrm{UO}_{2}$ fuel at 0 and $100 \%{ }^{2} \mathrm{H}$.

The Bonn-B $\Delta k_{\text {eff }}$ values from figure 1 are compared with similar values derived from table 2 in figure 2 . The spherical benchmark results are about six times more sensitive to the ${ }^{2} \mathrm{H}$ data library, changing by a total of about $1.2 \mathrm{mk}$ from 0 to $100 \%{ }^{2} \mathrm{H}$. This behaviour may be related to the harder spectrum of the spherical $\mathrm{UO}_{2}$ benchmark as shown in figure 3.

The changes in the relative neutron flux spectra (each spectrum is normalized to unity) between the Bonn-B and ENDF/B-VII.0 results have certain similarities for the $\mathrm{UO}_{2}$ sphere and 28-element $\mathrm{UO}_{2}$ fuel, depending on the ${ }^{2} \mathrm{H}$ concentration used, as shown in figure 4 . In general, the neutron flux spectrum is softer when the Bonn-B, ENDF/B-VI.4 or JENDL-3.3 data libraries for ${ }^{2} \mathrm{H}$ are used instead of the ENDF/B-VII.0 library, with the flux increasing below about $0.1 \mathrm{MeV}$ and decreasing above; these changes are larger for ZED- 2 cases with $\mathrm{D}_{2} \mathrm{O}$-cooled fuel channels and $\mathrm{UO}_{2}$-sphere cases having $100 \%{ }^{2} \mathrm{H}$. Although the differences between the Bonn-B and ENDF/B-VII.0 relative flux spectra for the $\mathrm{UO}_{2}$ sphere and 28-element $\mathrm{UO}_{2}$ fuel are similar in figure 4 , the 


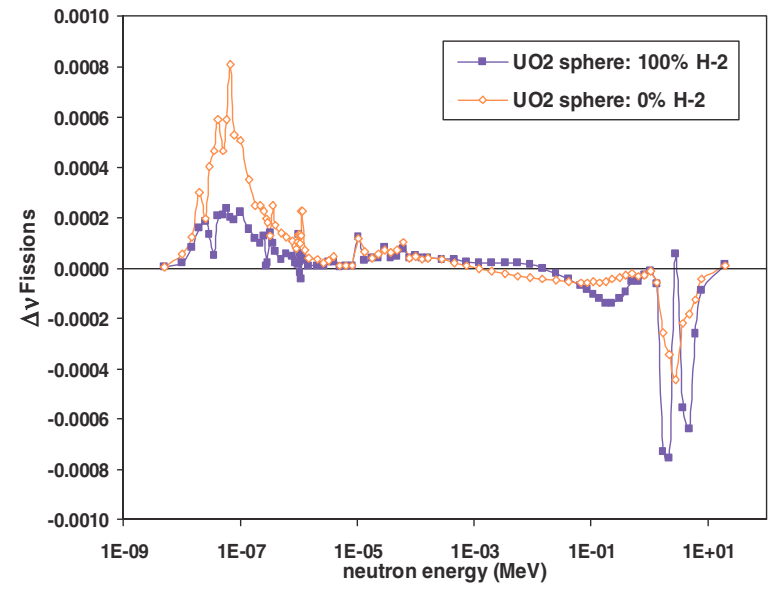

Fig. 6. Change in fission neutron yields between Bonn-B and $\mathrm{ENDF} / \mathrm{B}-\mathrm{VII} .0^{2} \mathrm{H}$ data for $\mathrm{UO}_{2}$ sphere at 0 and $100 \%{ }^{2} \mathrm{H}$.

contrast between the results for 0 and $100 \%{ }^{2} \mathrm{H}$ coolant is greater for the former.

The reactivity consequences induced by these spectral shifts are evaluated by considering the change in fission neutron yields (i.e., $v$ Fissions) for the 28-element $\mathrm{UO}_{2}$ fuel in figure 5 (with ZEEP booster rods) and for the $\mathrm{UO}_{2}$ sphere in figure 6.

The differences in fission yields at neutron energies $<1 \mathrm{MeV}$ are almost entirely due to changes associated with ${ }^{235} \mathrm{U}$ fissions and those $>1 \mathrm{MeV}$ with ${ }^{238} \mathrm{U}$ fissions. Net positive reactivity arises from the dominance of positive changes at thermal energies $<1 \mathrm{eV}$ over negative changes $>1 \mathrm{MeV}$. In both figures 5 and 6 , the positive component decreases and the negative component is more pronounced for $100 \%{ }^{2} \mathrm{H}$, in agreement with the $\Delta k_{\text {eff }}$ trends in figures 1 and 2 .

For the ZED-2 28-element $\mathrm{UO}_{2}$ results in figure 5, the oscillations observed at thermal energies $<1 \mathrm{eV}$ reflect the statistical uncertainties in the MCNP5 tallies, which are significant even after tracking 120 million active neutron histories. Additionally, the corresponding changes in fission yields in the booster fuel rods tend to obscure and cancel the positive reactivity component from the 28-element $\mathrm{UO}_{2}$ fuel at low energies. In contrast, the trends in the results for the simple $\mathrm{UO}_{2}$ sphere model in figure 6 are relatively clear after only 60 million active neutron histories and are more pronounced.

\section{Conclusions}

A notable observation from this work is the reduction of about $0.4 \mathrm{mk}$ in the MCNP5 ZED-2 CVR calculation bias that is obtained when the $\mathrm{O}$-in- $\mathrm{UO}_{2}$ thermal scattering data available with ENDF/B-VII.0 are used. A main conclusion is that the simplified spherical benchmark calculations qualitatively confirm corresponding ZED-2 results - namely the subtle difference in the reactivity sensitivity to the ${ }^{2} \mathrm{H}$ nuclear data files when ${ }^{2} \mathrm{H}$ "coolant" is mixed in with the $\mathrm{UO}_{2}$ fuel compared with the sensitivity when it is absent, and provide clarification by magnifying the underlying phenomena. Such corroboration implies that the ZED-2 $\mathrm{D}_{2} \mathrm{O}$ coolant-voidreactivity measurements appear capable of high reactivity sensitivity - to well within $\pm 1 \mathrm{mk}$. Thus, MCNP simulations of ZED-2 measurements will provide a good test of any revised $(n, d)$ energy-angle probability distributions. The new Bonn-B $(n, d)$ data tested in this work lend support to the earlier ENDF/B-VI.4 evaluation and, in the absence of precise new $(\mathrm{n}, \mathrm{d})$ scattering measurements, constitute a promising basis for a revision to the existing ${ }^{2} \mathrm{H}$ data library in ENDF/B-VII.0.

The author thanks R. Arcilla of NNDC for producing the ENDF/ BVII.0 ACE files, R.D. Mosteller of LANL for providing the ENDF/ B-VI. $4{ }^{2} \mathrm{H}$ ACE file and J.P. Svenne of the University of Manitoba and, especially, L. Canton of Padova University for generating the Bonn-B (n,d) scattering data tested in this study.

\section{References}

1. R.D. Mosteller, J.M. Campbell, R.C. Little, Reactivity Impact of ENDF/B-VI Cross Sections for Deuterium in Heavy-Water Solution Benchmarks, LA-UR-05-0330, Annual Meeting of the American Nuclear Society (ANS), June 5-9, 2005, San Diego, CA.

2. R.D. Mosteller, K.S. Kozier, J.M. Campbell, R.C. Little, Reactivity Impact of Deuterium Cross Sections for HeavyWater Benchmarks, LA-UR-05-0787, in Proceedings of the International Topical Meeting on Mathematics and Computation, Supercomputing, Reactor Physics, and Nuclear and Biological Applications, Avignon, France, Sept. 12-15, 2005.

3. X-5 Monte Carlo Team, MCNP - A General Monte Carlo NParticle Transport Code, Version 5, LANL report LA-UR-031987 (April 2003).

4. K.S. Kozier, Sensitivity of MCNP5 Calculations for a Spherical Numerical Benchmark Problem to the Angular Scattering Distributions for Deuterium, in Proceedings of PHYSOR-2006: Advances in Nuclear Analysis and Simulation, ANS Topical Meeting, Vancouver, BC, Canada, Sept. 10-14, 2006.

5. J.P. Svenne et al. (these proceedings).

6. M.B. Chadwick, P. Oblozinsky et al. (CSEWG collaboration), ENDF/B-VII: Next Generation Evaluated Nuclear Data Library for Nuclear Science and Technology, Nucl. Data Sheets 107, 2931 (Dec. 2006).

7. R.E. MacFarlane, D.W. Muir, The NJOY nuclear data processing system, version 91, LANL Report LA-12740-M, 1994. 